\title{
Nuclear deformation and anchorage defect induced by DCM mutants in $\operatorname{lamin} \mathrm{A}$
}

Manindra Bera ${ }^{1,3 \pi}$, Rinku Kumar² ${ }^{2 \pi}$ Bidisha Sinha ${ }^{2}$ and Kaushik Sengupta1,4*

2 Department of Biological Sciences, Indian Institute of Science Education and Research Kolkata, Mohanpur-741246, India

1.Biophysics \& Structural Genomics Division, Saha Institute of Nuclear physics, Kolkata-700064, India

3 Present address: Department of Cell Biology, Yale School of Medicine, New Haven, CT, USA 06510

4 Homi Bhabha National Institute, Mumbai-400094, India

If These authors contributed equally and the first author was selected on alphabetical order.

Running title: Nuclear deformations in cardiomyopathic nuclei

Keywords: Deformation; Stress; Cardiomyopathic; Nuclei

*To whom correspondence may be addressed

Kaushik Sengupta

Biophysics \& Structural Genomics Division, Saha Institute of Nuclear Physics, 1/AF, Bidhannagar, Kolkata-700064, India,

Phone- 03323375345 (Ext. 3504)

kaushik.sengupta@saha.ac.in 
Nuclear deformations in cardiomyopathic nuclei

ABSTRACT

Dilated Cardiomyopathy (DCM) is one of the different types of laminopathies caused by the mutations in A-type lamins in somatic cells. The involuntary cyclic stretching of cardiac muscle cells, as observed in normal physiological conditions is perturbed in DCM which afflict patients globally. As A-type lamins are principal components in nuclear mechanics, we have investigated the effect of the DCM causing mutants- K97E, E161K and R190W on nuclear stretching and deformation by static and dynamic strain inducing experiments. All the mutants exhibited differential nuclear structural aberrations along with a tilt in the nuclear axis compared to the direction of the cell axis and a significant decrease in the lamina thickness which reflected the lower mechanical rigidity. These phenotypes could potentially

11 lead to defects in nuclear anchorage to the actin filaments thereby resulting in the misshapen

12 and misaligned nucleus.

\section{INTRODUCTION}

Nuclear lamins which are type $\mathrm{V}$ intermediate filament proteins were first visualized

17 to form $\sim 50 \mathrm{~nm}$ meshwork underlying the inner nuclear membrane (1). It was considered

18 that lamin A forms 10-nm filaments inside the cell (2-5) until recently when this notion has

19 been revised by the cryo-ET structure of vimentin null mouse embryonic fibroblast

20 suggesting that both A- and B-type lamins form tetrameric $3.5 \mathrm{~nm}$ thick filaments inside the

21 nucleus (6). Earlier, it was also reported by the structured illumination microscopy that both

22 lamins form distinct meshwork in the nucleoplasm (7). Lamin A is a principal regulator of

23 nuclear mechanics and the relative abundance of the lamin A is dependent on the tissue types

24 and matrix elasticity $(8,9)$. More than 450 mutations have been discovered in the $L M N A$ gene

25 (http://www.umd.be/LMNA/) which produces almost 16 different types of diseases like

26 Dilated Cardiomyopathy (DCM), Emery-Dreifuss Muscular Dystrophy (EDMD) etc. that are

27 collectively coined as laminopathies (10-12). These are tissue-specific in nature

28 predominantly affecting muscle and adipose tissues. The hallmark of laminopathies is the

29 formation of misshapen and even fragile nuclei (13). Two hypotheses are in vogue to explain

30 the role of mutant lamin A protein in the pathogenesis of laminopathies. The gene regulation 
Nuclear deformations in cardiomyopathic nuclei

31 hypothesis correlated the occurrence of aberrant gene expression due to differential

32 transcriptional regulation by lamin A while the structural hypothesis focuses on the

33 perturbation effect of mutant lamin A on higher order assembly of the nuclear lamina leading

34 to fragile nuclei $(14,15)$. Previously, several reports based on AFM indentation, micropipette

35 aspiration and microrheological experiments showed altered nuclear elasticity for lamin A

36 null MEF or cells containing lamin A mutations (16-18). The rapidly growing area of

37 nucleoskeleton-cytoskeleton interactions has led to numerous studies involving the

38 transduction of external mechanical cues to the nucleus. Earlier studies revealed that

39 cytoskeleton maintains the nucleus in a pre-stressed state by aligned actin filaments forming

40 a perinuclear actin cap (19-21). Recently, it has been shown for endothelial cells that central

41 and apical stress fibers play distinct mechanical roles in maintaining coordination between

42 the cell and nuclear shapes (20).

43 In this report, we studied the effect of nuclear morphology due to dilated cardiomyopathic

44 mutations (K97E, E161K, and R910W) under different physiological strains. These

45 mutations were selected based on their severities of phenotypes in patients. The phenotype

46 of this disease is characterized by the cardiac arrhythmia with acute conduction defects and

47 myocardial infarction that can lead to sudden death (22). We took two different approaches

48 to observe the effect of the laminopathic mutations (human origin) in the mouse myoblast

49 C2C12. Cells were stretched by applying cyclic strain (dynamic strain) on the PDMS

50 membrane to mimic the physiological state of extension and relaxation of muscle and cardiac

51 cells. Secondly, cells were grown on the different micropatterned surfaces which exert static

52 deformation force (static strain) on the cytoskeleton via cell adhesion molecules $(23,24)$ and

53 ultimately perturbs the lamin A network assembly. In both the cases, we observed

54 differential nuclear deformations, anchorage defect characterized by a tilt in nuclear axis

55 about actin axis and variation in the lamina thickness which suggested a reduction in nuclear

56 rigidity and integrity. These results also suggest a probable explanation behind the

57 formation of elongated and misshapen nuclei in cardiomyopathy caused due to the LMNA

58 mutations. 
Nuclear deformations in cardiomyopathic nuclei

\section{RESULTS AND DISCUSSION}

\section{Differential impairment of the nucleo-actin axis on the micropatterned substrate}

63 The actin cytoskeleton is known to mechanically couple with the nuclear lamins through

64 Linker of Nucleoskeleton and Cytoskeleton (LINC) complexes (25). We measured the

65 alignment of the nucleus with the cell's orientation vector in cells expressing WT and mutant

66 Lamins A/C. Usually, the orientation vector of the cell reflects closely the orientation of actin

67 stress fibers (26). However, well-spread cells often had random orientations of stress fibers

68 as is evident from cells on non-patterned glass (Fig. 1 A, left). To be able to control this

69 variation, we used substrates in which the adhesion area was patterned by photomasks (Fig.

$701 \mathrm{~A}$, right). Two rectangular patterns denoted by RA and RB with aspect ratios 3, 2 and spread

71 areas $1200,450 \mu \mathrm{m}^{2}$ respectively, were chosen. These aspect ratios were chosen based on a

72 similar ratio (1:3) (27)of hPSC-derived monolayers of cardiomyocytes. To quantify the

73 alignment of the nucleus with the cell, the cell and nucleus outlines were fitted with ellipses

74 and the absolute angle between their major axes noted as orientation angle (Fig. $1 \mathrm{~B}$ ) for cells

75 expressing the GFP tagged versions of lamin A (Fig. 1 C). For perfect alignment of the nucleus

76 with the cell axis, the expected orientation angle is $0^{\circ}$, while for randomly oriented nuclei,

77 the angles are expected to vary from $0^{\circ}$ to $90^{\circ}$, therefore averaging at $45^{\circ}$ (dashed line, Fig.

$781 \mathrm{D})$. As suspected, the non-uniform shapes in non-patterned cells resulted in angles $\left(\sim 21^{\circ}\right)$

79 implying low alignment (Fig. 1 D). There was also no difference in orientation angles in

80 mutant lamins compared to wild type lamin A (denoted by WT) (Fig1 D). However, using

81 micropatterns, we first observed lowered angles $\left(\sim 10^{\circ}\right)$ for WT as expected from the

82 uniformity of the shape (Fig. 1 D). Secondly, we found that all mutants exhibited the

83 alignment of nucleus axis to cell axis within the range $24^{\circ}-37^{\circ}$ (Fig. 1 D, Supporting Table1)

84 rendering it closer to the value expected from random orientation $\left(45^{\circ}\right.$, Fig. $1 \mathrm{D}$, dashed line).

85 However, no particular parameter of nuclear shape (eccentricity, aspect ratio, circularity, -

86 Fig. 1 D) was significantly affected by the mutations. This strongly suggested the lack of

87 nuclear alignment to cell-axis presumably due to a loss of mechanical coupling between the

88 nucleus and cytoplasmic actin network (Fig. 1 D, top). Furthermore, we observed the lamin

89 A aggregation inside the nucleoplasm and quantified the size in terms of area. K97E and

90 R190W transfected nuclei showed significantly larger aggregates, $\sim 14$ and $12 \mu \mathrm{m}^{2}$ 
Nuclear deformations in cardiomyopathic nuclei

91 respectively. WT and E161K nuclei showed smaller aggregates compared, $\sim 2.5$ and $8.3 \mu \mathrm{m}^{2}$ 92 respectively on the RB micropatterned surface. But on the RA pattern, E161K and R190W 93 nuclei produced smaller aggregates compared to $\mathrm{RB}, \sim 4.6$ and $5.5 \mu \mathrm{m}^{2}$ respectively (shown

94 in Supplementary Fig1). The size of aggregates for WT and K97E were unchanged, 2 and $9515 \mu \mathrm{m}^{2}$ respectively. The aggregate may constitute other subtypes of endogenous lamin also 96 because of its homo- and hetero-polymerization nature but we only monitored GFP97 fluorescence of the exogenous lamin A. Since all these experiments were performed by 98 transient transfection, that enhance the possibility of overexpression of protein lead to 99 misfolded protein and aggregation. We ruled out this possibility by measuring the non100 significant change in the amount of lamin upon transfection through western blotting 101 (shown in Supplementary Fig 3).

\section{Nuclear deformations after the cell stretching}

103 In cardiac muscle tissues, the cells continuously experience the cyclic and uniaxial stretching.

104 Hence, the cells also reorient themselves towards the stretching axis $(28,29)$. Zimmermann 105 et al. stretched neonatal rat heart cells up to $10 \%$ at $2 \mathrm{~Hz}$ frequency to generate engineered 106 heart tissue (30) and Yu et al. showed that 10\% static stretching can insert new sarcomere 107 in the neonatal rat (31). The normal resting heart rate in infant and in the case of tachycardia 108 in adult heart rate can be more than $2 \mathrm{~Hz}(32,33)$. We intended to test the impact of chronic 109 mechanical perturbation (cyclic stretching) on lamin A-dependent nuclear morphology by 110 mimicking physiological frequency of extension and relaxation of muscles and cardiac cells. We

111 performed the mechanical stretching experiments on the wild-type (WT) and DCM-causing 112 mutant lamin A transfected C2C12 cells. The cells were grown on the PDMS membrane and 113 the membrane was stretched cyclically up to $10 \%$ for $2.5 \mathrm{hr}$. at a frequency of $2 \mathrm{~Hz}$. We 114 measured how the mutants differed from the WT in shape parameters and orientation of 115 nucleus with and without cyclic stretching. In the absence of cyclic stretch, WT and mutant 116 cells grown on PDMS displayed non-significant differences in most (8/12) parameters 117 except for the observations that R190Wwas less aligned than WT while E161K resulted in 118 nuclei with higher eccentricity and aspect ratio and lower circularity. However, after cyclic 119 stretch was imparted most (10/12) parameters were found to be different - especially the 120 ones quantifying nuclei shape changes. Eccentricity and aspect ratio of all three mutants 121 increased on stretching in contrast to E161K which showed an increase even in the absence 
Nuclear deformations in cardiomyopathic nuclei

122 of stretching. Correspondingly, the circularity of all three mutants decreased on cyclic

123 stretching in contrast to only E161K showing a decrease in the absence of stretching. It must

124 be emphasized that, K97E showed a tendency to deviate from the WT in mechanically

125 unperturbed condition (Fig. 2). On cyclic stretching nuclear deformation of K97Ewas seen to

126 largest among all mutants. Thus, we quantified alignment of the nucleus with the cell body

127 (orientation angle difference) by employing micropatterning to reduce the initial

128 heterogeneity of cell shape in the population (reduced standard deviation of $\theta$, Supporting

129 Table 1). Here, we could elucidate that lamin A mutations may lead to weaker coupling of the

130 nucleus shape to the cell's shape (Supporting Table 2). Next, we demonstrated that when

131 these mutants undergo mechanical perturbation, they are less resistant to shape changes

132 and therefore underwent deformations, unlike WT nuclei that did not show any significant

133 alteration in nucleus-to-cell alignment or shape on cyclic stretching (Supporting Table 3).

134 We have calculated the meshwork size of lamin A for WT and R190W (Supplementary Fig 2).

135 R190W nuclei dilated lamin A meshwork compared to the WT-nuclei which reflected the

136 lower mechanical rigidity of the nuclei. K97E and E161K nuclei produced lamin A aggregates

137 only.

\section{Reduction in the lamina thickness due to laminopathic mutation}

139 Nuclear stiffness is primarily determined by nuclear lamina meshwork. We measured lamina

140 thickness which could be the direct readout of stiffness as reported earlier as well (34). We

141 assessed the impact of laminopathic mutations on nuclear lamina thickening behavior. We

142 also included the effect of spreading as reported earlier that lowering in the spread area

143 reduces the stress fiber driven compression of the nucleus and nuclear volume which in turn

144 reduces physical strain on the nucleus (35). We chose two spread area (RA,1200 $\mu^{2}$ and

$145 \mathrm{RB}, 450 \mu \mathrm{m}^{2}$ ) which has $\sim 3$-fold spread area difference. We measured the lamina thickness

146 by structured illumination microscopy (SIM) whose lateral resolution was calculated to be

$147 \sim 120 \mathrm{~nm}$ (shown in Supplementary Figure 3). We measured average thickness $0.35 \pm 0.03$

$148 \mu \mathrm{m}$ of the WT lamina on the RA patterned that reduces to $0.30 \pm 0.03 \mu \mathrm{m}$ on RB surface

149 (shown in Fig. 3 A-D, left) suggesting thickening of the lamina on highly spread cells. We

150 believed that lamina in the low spread area cells is less stiff thereby resulting in the less

151 aligned network and showed lamina softening behavior. On comparing lamina thickness of 
Nuclear deformations in cardiomyopathic nuclei

152 WT with R190W and E161K transfected nuclei, we observed that the lamina thickness did 153 not alter for wild-type $(\sim 0.3 \pm 0.05 \mu \mathrm{m})$ and R190W $(\sim 0.29 \pm 0.02 \mu \mathrm{m})$, however, E161K 154 mutant lamina $(\sim 0.23 \pm 0.03 \mu \mathrm{m})$ showed significant softening behavior compared to WT on 155 the RB surface (Figure 3D). But on RA, the lamina for both E161K and K97E were not clearly 156 visible and both the nuclei showed a significantly large number of aggregates. All the values 157 are tabulated in the supporting table 3. The effect of the geometrical constraint on the K97E158 lamin A transfected nuclei were similar. In both cases (RA \& RB) for K97E nuclei, the lamina 159 was not clearly visible and nuclei showed larger aggregates. On the spread cell area (RA), the 160 mechanical strain perturbed the lamin A assembly for the mutant nuclei, hence, lamina 161 thickness was significantly decreased or abolished. This lamina softening behavior might 162 explain the lower mechanical rigidity of the nuclei among the cardiomyopathic patients.

\section{CONCLUSION}

164 In this article, we studied the nuclear deformation and misalignments of the nuclear axis 165 compared to the cell axis under different physiological strain. We chose different DCM 166 causing LMNA mutants (K97E, E161K, and R190W) and applied external mechanical strain 167 to mimic the physiological condition. In addition, we measured the variation in lamina 168 thickness which plausibly explained the origin of DCM disease and its severity. We observed

169 all the mutations produced significant nuclear deformations and showed significant 170 misalignments of nuclear axes when cyclic stretching was applied. On different 171 micropatterned surfaces, K97E and E161K nuclei produced significantly large lamin A 172 aggregates. Previously, it was reported that no apparent changes in the nuclear lamina 173 occurred for E161K mutation in heart tissue (36). As similarly overexpressed WT nuclei 174 showed less or no visible aggregates, hence the aggregation in the mutant nuclei was due to 175 the overexpression of the improperly assembled mutants lamin A. Previously, it has also 176 been reported that the perinuclear actin and TAN lines anchor the nucleus in pre-stressed 177 condition and help to maintain its orientation (37) and nesprin-1 (an important LINC 178 partner) also helps in tethering the nucleus to actin in C2C12 cells (38). A plausible 179 explanation for impairment of nucleo-actin axis might be the progressive weakening of 180 lamina at points such that the contact nodes with the actin filaments are abrogated thereby 181 resulting in impairment about the axis of the TAN lines. It is important to note that we had 
Nuclear deformations in cardiomyopathic nuclei

182 previously observed bundling behavior in E161K \& R190W which lead to misshapen nuclei 183 with reduced viscoelastic properties. But in the case of the K97E, the lamin A network 184 formation was significantly altered which was reflected on the nuclear elasticity $(39,40)$. 185 Therefore, the cell might not respond to the external mechanical cues effectively which in 186 turn can lead to altered cell response. This might be the reason behind significant nuclear 187 deformations under cyclic stretching. Furthermore, we studied the variation in lamina 188 thickness by SIM. The thickness of the lamina is in the range of $\sim 20-50 \mathrm{~nm}$ and electron 189 microscopic study revealed that lamina thickness can vary from $\sim 18 \mathrm{~nm}$ to $100 \mathrm{~nm}$ in human 190 cartilage due to injury(41). Earlier, Schermmelleh et al. showed by 3D-SIM that thickness of 191 the lamin B1 thread is $\sim 100 \mathrm{~nm}$ laterally and $\sim 300 \mathrm{~nm}$ axially in C2C12 cells (because of its 192 resolution limit) (42). But in our experiments, lamina thickness was detected around $\sim 0.3$ $193 \mu \mathrm{m}$ which could be due to both overexpression of lamin A and response to mechanical stress. 194 But within the resolution limits $(\sim 120 \mathrm{~nm})$ of our experiments, we confirmed the thickening 195 behavior of lamina. We observed that WT lamina thickness slightly increased on the 196 geometrically constrained surface area. But the mutant nuclei showed drastic effect. We 197 could not detect any rim staining of lamin A on the RA surface for the E161K mutant, instead, 198 they formed larger aggregates in the nucleoplasm. The lamina thickness for the R190W 199 mutant nuclei also decreased when cells were grown on the RB surface. We also observed 200 that the total lamin A expression did not change because of the mutation (Supplementary Fig 201 3). These results suggested that soluble lamin A might get recruited to the lamina in response 202 to an increase in the mechanical strain, but mutant lamin A showed the defect in the lamina 203 assembly. Therefore, R190W nuclei showed less thick lamina and E161K nuclei showed 204 larger lamin A aggregates in the nucleoplasm. Due to mechanical stress, changes in the lamin 205 A structure and the phosphorylation status may lead to a change in the lamina organization $206(43,44)$. It is already established that at higher mechanical stress, lamin A accumulates at the 207 nuclear periphery that leads to the increment in nuclear stiffness and at low strain, changes 208 in the phosphorylation status produce more soluble lamin A. Hence, lamin A follows a 209 'mechanostat equilibrium' in the nucleoplasm (45). The change in lamina thickness could be 210 due to the accumulation of the lamin $\mathrm{A}$ in the aggregates and the change in the 211 phosphorylation pattern. The increase in WT lamina thickness due to the increase in 
Nuclear deformations in cardiomyopathic nuclei

212 mechanical stress suggested that changes in the phosphorylation profiles led to the

213 accumulation of the insoluble lamin A to the envelope. But the mutant nuclei lack this ability

214 leading to improper self-assembly and aggregate formation. Ectopic expression of lamin A in

215 the background of the endogenous lamins can significantly contribute to the nuclear stiffness

216 (46). But the background lamin expression was not sufficient to resist the lamina

217 deformation due to different mechanical strain. The aspect ratio of the micropattern was like

218 the hPSC-derived monolayer of the immature cardiomyocytes. The change in the nuclear

219 organization due to the geometrical constraint might be irreversible in the case of

220 cardiomyocytes which might lead to laminopathies. Based on our experimental findings

221 which we have summarized all the results in Supporting Table 1, 2 and 3. We hereby propose

222 that external mechanical cue can alter lamin A meshwork density significantly in presence

223 of laminopathic mutations; lamina shows stress induced thinning behavior and soluble lamin

224 A forms large insoluble aggregates inside the nucleoplasm. These factors cumulatively affect

225 the nuclear rigidity which alters the nuclear shape and mechanics. A plausible model for

226 occurring of laminopathy diseases due to single-point mutation is shown in Fig 4 . In all

227 cases, we noticed differential alterations in the nuclear shape and the lamin A meshwork for

228 the DCM mutations. The mutant nuclei produced a severe deformation when exposed to the

229 external mechanical strain. In the physiological state also, we can predict that mutation of

230 lamin $\mathrm{A}$ in the tissues which are continuously exposed to the external strain can produce

231 significant damage to the nuclear shape and lamin meshwork. Eventually, that can lead to

232 differential gene expression programme and thereby produce the disease. 
Nuclear deformations in cardiomyopathic nuclei

\section{METHODS}

\section{Site-directed mutagenesis}

243 All the mutations were generated using site-directed mutagenesis methods in EGFP-LA plasmid and

244 the details of the primer are reported in Bhattacharjee et al. (40). The mutations were confirmed by

245 the Sanger sequencing method(47).

\section{Micropatterning glass coverslip}

24722 x $22 \mathrm{~mm}$ glass coverslips were etched in ethanol: acetic acid (19:1) mixture for $30 \mathrm{~min}$, following 248 by ethanol washing and air drying. Dried coverslips were treated with UV Ozone cleaner (Jelight 249 Company, USA) for $5 \mathrm{~min}$ and incubated with $0.2 \mathrm{mg} / \mathrm{ml}$ PLL-g-PEG (SuSos, Switzerland) solution 250 (prepared in $10 \mathrm{mM}$ HEPES buffer, $\mathrm{pH}$ 8.3) for $1 \mathrm{~h}$. Photo-masks (JD, Photo Data, UK) is cleaned with 251 acetone and isopropanol followed by 5 min UV Ozone cleaning. PEG-coated coverslips are attached 252 on the chrome side of already cleaned photo-masks with the help of a drop of water. Excess water 253 was soaked with tissue paper for firm adherence of coverslip to mask. Coverslips attached with 254 photo-mask were illuminated with deep UV for $5 \mathrm{~min}$ by placing non-chrome side facing UV lamp. 255 Patterned coverslips were detached by floating into water followed by 45 min incubation in $20 \mu \mathrm{l}$ 256 fibronectin solution (Sigma) $\left(25 \mu \mathrm{g} / \mathrm{ml}\right.$ solution in $\mathrm{NaHCO}_{3}$, pH 8.6). Finally, patterned coverslips 257 were used for cells seeding.

\section{Transfection and cells seeding}

$25970 \%$ of confluence C2C12 cells were transfected with $3 \mu$ g GFP lamin mutants by lipofection 260 (Lipofectamine 3000, Life Technologies) following manufacturer instructions. Post $16 \mathrm{~h}$ of 261 transfection, cells were detached from culture dish using $0.02 \%$ EDTA solution (Calbiochem, USA) 262 prepared in cell culture grade PBS (Sigma). $2 \times 10^{5}$ cells were seeded on each patterned coverslips 263 and unattached cells washed off after 30 using warm media. For cell stretching, $10^{5}$ cells per $500 \mu \mathrm{l}$ 264 culture media on each PDMS sheet were seeded. After transfection, Lamin A expression was checked 265 mouse monoclonal anti-lamin A/C antibody at 1:1000 dilution.

\section{Cell Stretching}

267 Custom made cells stretching device was employed for cell stretching as used earlier (ref). Briefly, 268 Silicon elastomer (SYLGRAD 184, Dow Corning) and the curing agent was mixed in 10:1 proportion, 269 an air bubble was removed by centrifuging $5 \mathrm{~min}$ at $3000 \mathrm{rpm} .3 \mathrm{ml}$ mixture was spread over each

$27090 \mathrm{~mm}$ dish. Placed vertically to get excess mixture flow away. The dish at this configuration was 
Nuclear deformations in cardiomyopathic nuclei

271 baked at $60^{\circ} \mathrm{C}$ to get $100 \mu \mathrm{m}$ thick PDMS sheet. PDMS sheet was treated $0.5 \mathrm{mg} / \mathrm{mlSulfo}-\mathrm{SANAPAH}$

272 (Pierce chemicals) under deep UV for 5 min (UVO cleaner, Jelight, Inc., USA), functionalized with

273 fibronectin (25 $\mathrm{\mu g} / \mathrm{ml}$ solution in $\mathrm{NaHCO}_{3}$, pH 8.6 Sigma). Fibronectin functionalized coverslips were

274 mounted and custom builds stretchers, GFP Lamin transfected cells were seeded on $25 \times 25 \mathrm{~mm}$ area

275 encircled with silicon grease. Cells were grown for $24 \mathrm{~h}$ on PDMS loaded stretcher inside the

276 incubator. For cyclic cell stretching, PDMS sheet containing C2C12 cells grown in a well was attached

277 to two motors (Physik Instrumente (PI), GmbH and Co KG) using custom-designed adapters. Cyclic

278 stretching at $2 \mathrm{~Hz}$ frequency and 10\% amplitude was performed with MATLAB for $2.5 \mathrm{~h}$. Stretching

279 was performed inside the cell-culture incubator.

\section{Cell fixation and microscopy}

281 C2C12 cells either grown on glass or PDMS sheet were fixed using 4\% PFA on their respective 282 surfaces for $12 \mathrm{~min}$, following PBS wash. Cells were permeabilization by $0.2 \%$ Triton X-100 solution 283 (Sigma) in PBS, stained with DAPI (2.8 $\mu \mathrm{M}$, Sigma) and Phalloidin Alexa Fluor $568(0.35 \mu \mathrm{M}$,

284 Molecular probe, Life Technologies) for $2 \mathrm{~h}$ at room temperature. Finally, coverslips were mounted 285 on with slides (Sigma) for imaging. In case of cells grown on a PDMS sheet, a number one glass 286 coverslips were first attached on top of the PDS sheet with mounting media (Mowiol, Sigma). The 287 entire stretching device was flipped upside down and captured images using oil objective through 288 the glass. Z-stack ( $0.5 \mu \mathrm{m}$ step size) images were captured by Olympus epi-fluorescence microscope 289 (Olympus Corporation Japan) with 100X, 1.49NA objective and sCMOS camera (Orca-Flash 4.0, 290 Hamamatsu Photonics Japan) with pixel size $65 \mathrm{~nm}$.

\section{Nucleus to cell orientation angle difference and nucleus shape parameter extraction}

292 Image analysis was done using software ImageJ/Fiji ((https://imagej.net/Fiji)). Nucleus (GFP lamin) 293 to cell (actin) orientation angle difference was measured in two-parts, first drawing ROI manually on 294 maximum intensity projection image to outline object, second fitting ROI to an ellipse for measuring 295 orientation angle. Major (D1) and minor (D1) axis of the fitted ellipse on the nucleus was used for

296 eccentricity $\left(\sqrt{\left(1-\frac{D 2}{D 1}\right)^{2}}\right.$ ) and aspect ratio (D1/D2) measurement. Perimeter and area of object

297 (nucleus) outline on maximum intensity projection image were used for circularity $\left(4 \pi \times \frac{\text { Area }}{\text { Perimeter }^{2}}\right)$ 298 measurement.

299 Mesh size and width of the peripheral lamin measurements 
Nuclear deformations in cardiomyopathic nuclei

300 Cells were washed three times with ice-cold 1X PBS (pH 7.4) and fixed with 4\% paraformaldehyde.

301 Actin was stained with Alexa-561 conjugated phalloidin (Thermo Fisher Scientific) with 1:1000

302 dilutions. After proper washing with PBS, the coverslips were stained with Vectashield that contained

303 DAPI (Vector Laboratories). The coverslips were sealed with watercolor nail polish. The slides were

304 visualized with NIKON Inverted Research Microscope ECLIPSE TiE with Plan Apo VC 100X oil DIC N2

305 objective/1.40 NA/1.515 RI with a digital 4X zoom. Images were analyzed using Ni Elements AR Ver

3064.13 and Image J (Fiji). The X-Y resolution of N-SIM was calibrated to $120-150 \mathrm{~nm}$ using $20 \mathrm{~nm}$

307 FluoSphere beads (Thermo Scientific, F887) in $580 \mathrm{~nm}$ laser. We measured the mesh sizes using the

308 area selection tool of Ni-Elements software, but the lines were drawn considering the FWHM method

309 where lower values were considered as the limit of the sides. To measure the lamina thickness, first

310 nuclear rim staining of lamin A were straightened using Image J and then lines were drawn across

311 the linearized lamina and intensity profiles were plotted. From the intensity profile, FHWM was

312 considered to be the thickness or width of the lamina. The thickness of each nucleus was calculated

313 by averaging 10 randomly chosen line profiles and more than 15 separate nuclei were considered for

314 each condition. Sizes of aggregates were measured using an area selection tool of Ni-Elements. The

315 statistics were generated using Origin Pro 8.5 software.

\section{ACKNOWLEDGMENTS}

317 Authors thank Arikta Biswas for her generous help in the instrument set up.

318 FUNDING

319 The authors thank the Department of Atomic Energy and SERB, Department of Science \& Technology,

320 India for research Grant and fellowship. B. S. acknowledges the Wellcome Trust DBT-India Alliance

321 (IA/I/13/1/500885) for financial support.

\section{AUTHORS CONTRIBUTION}

323 KSG, BS, MB \& RK designed all the experiments. RK \& MB performed all the experiments. KSG, MB,

324 RK \& BS analyzed the data and wrote the paper. KSG conceived the entire project and responsible for 325 finances related to the project.

\section{COMPETING FINANCIAL INTERESTS}

327 The authors declare no competing financial interests. 


\section{Nuclear deformations in cardiomyopathic nuclei}

\section{0}

3311.

332

333

334

335

336

337

338

339

340

341

342

343

344

345

346

347

348

349

350

351

352

353

354

355

356

357

358

359

360

361

362

363

364

\section{REFERENCES}

1. Fawcett, D. W. (1966) On the occurrence of a fibrous lamina on the inner aspect of the nuclear envelope in certain cells of vertebrates. The American journal of anatomy 119, 129-145

2. Heitlinger, E., Peter, M., Haner, M., Lustig, A., Aebi, U., and Nigg, E. A. (1991) Expression of chicken lamin B2 in Escherichia coli: characterization of its structure, assembly, and molecular interactions. The Journal of cell biology 113, 485-495

3. Strelkov, S. V., Kreplak, L., Herrmann, H., and Aebi, U. (2004) Intermediate filament protein structure determination. Methods in cell biology 78, 25-43

4. Stuurman, N., Heins, S., and Aebi, U. (1998) Nuclear lamins: their structure, assembly, and interactions. Journal of structural biology 122, 42-66

5. Dechat, T., Pfleghaar, K., Sengupta, K., Shimi, T., Shumaker, D. K., Solimando, L., and Goldman, R. D. (2008) Nuclear lamins: major factors in the structural organization and function of the nucleus and chromatin. Genes \& development 22, 832-853

6. Turgay, Y., Eibauer, M., Goldman, A. E., Shimi, T., Khayat, M., Ben-Harush, K., Dubrovsky-Gaupp, A., Sapra, K. T., Goldman, R. D., and Medalia, O. (2017) The molecular architecture of lamins in somatic cells. Nature 543, 261-264

7. Shimi, T., Kittisopikul, M., Tran, J., Goldman, A. E., Adam, S. A., Zheng, Y., Jaqaman, K., and Goldman, R. D. (2015) Structural organization of nuclear lamins A, C, B1, and B2 revealed by superresolution microscopy. Molecular biology of the cell 26, 4075-4086

8. Swift, J., Ivanovska, I. L., Buxboim, A., Harada, T., Dingal, P. C., Pinter, J., Pajerowski, J. D., Spinler, K. R., Shin, J. W., Tewari, M., Rehfeldt, F., Speicher, D. W., and Discher, D. E. (2013) Nuclear lamin-A scales with tissue stiffness and enhances matrix-directed differentiation. Science 341, 1240104

9. Harada, T., Swift, J., Irianto, J., Shin, J. W., Spinler, K. R., Athirasala, A., Diegmiller, R., Dingal, P. C., Ivanovska, I. L., and Discher, D. E. (2014) Nuclear lamin stiffness is a barrier to 3D migration, but softness can limit survival. The Journal of cell biology 204, 669-682

10. Capell, B. C., and Collins, F. S. (2006) Human laminopathies: nuclei gone genetically awry. Nature reviews. Genetics 7, 940-952

11. Bonne, G., Mercuri, E., Muchir, A., Urtizberea, A., Becane, H. M., Recan, D., Merlini, L., Wehnert, M., Boor, R., Reuner, U., Vorgerd, M., Wicklein, E. M., Eymard, B., Duboc, D., Penisson-Besnier, I., Cuisset, J. M., Ferrer, X., Desguerre, I., Lacombe, D., Bushby, K., Pollitt, C., Toniolo, D., Fardeau, M., Schwartz, K., and Muntoni, F. (2000) Clinical and molecular genetic spectrum of autosomal dominant Emery-Dreifuss muscular dystrophy due to mutations of the lamin A/C gene. Annals of neurology 48, 170-180

12. Raffaele Di Barletta, M., Ricci, E., Galluzzi, G., Tonali, P., Mora, M., Morandi, L., Romorini, A., Voit, T., Orstavik, K. H., Merlini, L., Trevisan, C., Biancalana, V., Housmanowa-Petrusewicz, I., Bione, S., Ricotti, R., Schwartz, K., Bonne, G., and Toniolo, D. (2000) Different mutations in the LMNA gene cause 
Nuclear deformations in cardiomyopathic nuclei

autosomal dominant and autosomal recessive Emery-Dreifuss muscular dystrophy. American journal of human genetics $\mathbf{6 6}, 1407-1412$

13. Ho, C. Y., Jaalouk, D. E., and Lammerding, J. (2013) Novel insights into the disease etiology of

14. Bertrand, A. T., Chikhaoui, K., Yaou, R. B., and Bonne, G. (2011) Clinical and genetic heterogeneity in

15. Worman, H. J., and Bonne, G. (2007) "Laminopathies": a wide spectrum of human diseases. Experimental cell research 313, 2121-2133

16. Radmacher, M. (2007) Studying the mechanics of cellular processes by atomic force microscopy. Methods in cell biology 83, 347-372

17. Lammerding, J., Dahl, K. N., Discher, D. E., and Kamm, R. D. (2007) Nuclear mechanics and methods. Methods in cell biology 83, 269-294

18. Rowat, A. C., Lammerding, J., Herrmann, H., and Aebi, U. (2008) Towards an integrated understanding of the structure and mechanics of the cell nucleus. BioEssays : news and reviews in molecular, cellular and developmental biology 30, 226-236

19. Khatau, S. B., Hale, C. M., Stewart-Hutchinson, P. J., Patel, M. S., Stewart, C. L., Searson, P. C., Hodzic, D., and Wirtz, D. (2009) A perinuclear actin cap regulates nuclear shape. Proceedings of the National Academy of Sciences of the United States of America 106, 19017-19022

20. Versaevel, M., Grevesse, T., and Gabriele, S. (2012) Spatial coordination between cell and nuclear shape within micropatterned endothelial cells. Nature communications 3, 671

21. Mazumder, A., Roopa, T., Basu, A., Mahadevan, L., and Shivashankar, G. V. (2008) Dynamics of chromatin decondensation reveals the structural integrity of a mechanically prestressed nucleus.

22. Taylor, M. R., Fain, P. R., Sinagra, G., Robinson, M. L., Robertson, A. D., Carniel, E., Di Lenarda, A., Bohlmeyer, T. J., Ferguson, D. A., Brodsky, G. L., Boucek, M. M., Lascor, J., Moss, A. C., Li, W. L., Stetler, G. L., Muntoni, F., Bristow, M. R., Mestroni, L., and Familial Dilated Cardiomyopathy Registry Research, G. (2003) Natural history of dilated cardiomyopathy due to lamin A/C gene mutations. Journal of the American College of Cardiology 41, 771-780

23. Garrison, M. D., McDevitt, T. C., Luginbuhl, R., Giachelli, C. M., Stayton, P., and Ratner, B. D. (2000) Quantitative interrogation of micropatterned biomolecules by surface force microscopy. Ultramicroscopy 82, 193-202

24. Lehnert, D., Wehrle-Haller, B., David, C., Weiland, U., Ballestrem, C., Imhof, B. A., and Bastmeyer, M. (2004) Cell behaviour on micropatterned substrata: limits of extracellular matrix geometry for spreading and adhesion. Journal of cell science 117, 41-52

25. Wang, N., Tytell, J. D., and Ingber, D. E. (2009) Mechanotransduction at a distance: mechanically coupling the extracellular matrix with the nucleus. Nat Rev Mol Cell Biol 10, 75-82 


\section{Nuclear deformations in cardiomyopathic nuclei}

401 26. Hatch, E. M., and Hetzer, M. W. (2016) Nuclear envelope rupture is induced by actin-based nucleus confinement. The Journal of cell biology 215, 27-36

27. Lundy, S. D., Zhu, W. Z., Regnier, M., and Laflamme, M. A. (2013) Structural and functional maturation of cardiomyocytes derived from human pluripotent stem cells. Stem cells and development 22, 19912002

28. Liau, B., Zhang, D., and Bursac, N. (2012) Functional cardiac tissue engineering. Regenerative medicine

29. Hirt, M. N., Hansen, A., and Eschenhagen, T. (2014) Cardiac tissue engineering: state of the art. Circulation research 114, 354-367

30. Zimmermann, W. H., Melnychenko, I., Wasmeier, G., Didie, M., Naito, H., Nixdorff, U., Hess, A., Budinsky, heart tissue grafts improve systolic and diastolic function in infarcted rat hearts. Nature medicine $\mathbf{1 2}$, $452-458$

31. Yu, J. G., and Russell, B. (2005) Cardiomyocyte remodeling and sarcomere addition after uniaxial static

32. Fleming, S., Thompson, M., Stevens, R., Heneghan, C., Pluddemann, A., Maconochie, I., Tarassenko, L.,

33. Pinto, D. S., Ho, K. K., Zimetbaum, P. J., Pedan, A., and Goldberger, A. L. (2003) Sinus versus nonsinus

34. Buxboim, A., Irianto, J., Swift, J., Athirasala, A., Shin, J. W., Rehfeldt, F., and Discher, D. E. (2017) Coordinated increase of nuclear tension and lamin-A with matrix stiffness outcompetes lamin-B receptor that favors soft tissue phenotypes. Molecular biology of the cell 28, 3333-3348

35. Kim, D. H., Li, B., Si, F., Phillip, J. M., Wirtz, D., and Sun, S. X. (2015) Volume regulation and shape bifurcation in the cell nucleus. J Cell Sci 128, 3375-3385

36. Mewborn, S. K., Puckelwartz, M. J., Abuisneineh, F., Fahrenbach, J. P., Zhang, Y., MacLeod, H., Dellefave, L., Pytel, P., Selig, S., Labno, C. M., Reddy, K., Singh, H., and McNally, E. (2010) Altered chromosomal positioning, compaction, and gene expression with a lamin A/C gene mutation. PloS one 5, e14342

37. Maninova, M., Iwanicki, M. P., and Vomastek, T. (2014) Emerging role for nuclear rotation and orientation in cell migration. Cell adhesion \& migration 8, 42-48

38. Espigat-Georger, A., Dyachuk, V., Chemin, C., Emorine, L., and Merdes, A. (2016) Nuclear alignment in myotubes requires centrosome proteins recruited by nesprin-1. Journal of cell science 129, 4227-4237 


\section{Nuclear deformations in cardiomyopathic nuclei}

438 40. Bhattacharjee, P., Banerjee, A., Banerjee, A., Dasgupta, D., and Sengupta, K. (2013) Structural alterations of Lamin A protein in dilated cardiomyopathy. Biochemistry 52, 4229-4241

440 41. Ghadially, F. N., Dick, C. E., and Lalonde, J. M. (1980) Thickening of the nuclear fibrous lamina in injured

42. Schermelleh, L., Carlton, P. M., Haase, S., Shao, L., Winoto, L., Kner, P., Burke, B., Cardoso, M. C., Agard, D. A., Gustafsson, M. G., Leonhardt, H., and Sedat, J. W. (2008) Subdiffraction multicolor imaging of the nuclear periphery with 3D structured illumination microscopy. Science 320, 1332-1336

43. Bera, M., Kotamarthi, H. C., Dutta, S., Ray, A., Ghosh, S., Bhattacharyya, D., Ainavarapu, S. R., and Sengupta, K. (2014) Characterization of unfolding mechanism of human lamin A Ig fold by singlemolecule force spectroscopy-implications in EDMD. Biochemistry 53, 7247-7258

44. Buxboim, A., Swift, J., Irianto, J., Spinler, K. R., Dingal, P. C., Athirasala, A., Kao, Y. R., Cho, S., Harada, T., Shin, J. W., and Discher, D. E. (2014) Matrix elasticity regulates lamin-A,C phosphorylation and turnover with feedback to actomyosin. Current biology : CB 24, 1909-1917

45. Osmanagic-Myers, S., Dechat, T., and Foisner, R. (2015) Lamins at the crossroads of mechanosignaling.

Genes \& development 29, 225-237

453 46. Zwerger, M., Jaalouk, D. E., Lombardi, M. L., Isermann, P., Mauermann, M., Dialynas, G., Herrmann, H., Wallrath, L. L., and Lammerding, J. (2013) Myopathic lamin mutations impair nuclear stability in cells and tissue and disrupt nucleo-cytoskeletal coupling. Human molecular genetics 22, 2335-2349 synthesis with DNA polymerase. Journal of molecular biology $\mathbf{9 4 , 4 4 1 - 4 4 8}$ 
Nuclear deformations in cardiomyopathic nuclei

\section{FIGURE LEGEND}

Figure 1. Lamin A mutations lead to the altered alignment of the nucleus with the cell axis. (A) Schematic representations depicting variations possible in the nucleus and cell shape along with nucleus alignment with cell axis in non-patterned (grown on glass) (left) and patterned cells (right). Ellipses are fits to cell-outline and nucleus-outline; the corresponding major axes represented as dashed lines. Wide-field transmission images of patterns on photomasks used to create micropatterns - RA (60 $\mu \mathrm{m} \times 20 \mu \mathrm{m})$ and RB $(30 \mu \mathrm{m} \times 15 \mu \mathrm{m})$ are shown here. Scale bar, $50 \mu \mathrm{m}$. (B) Schematic representation of cell to nuclear orientation angle ( $\theta$ ) and major (D1) and minor (D2) diameters of nucleus used for calculating nuclear eccentricity and aspect ratio. (C) Representative images of cells expressing GFP-Lamin A/C (green) mutants stained with DAPI (blue, DNA) and Phalloidin Alexa Fluor 568 (red, F-actin). Images show basal plane for actin and the maximum intensity projection for DAPI and GFP-Lamin A/C. Scale, $10 \mu \mathrm{m}$. (D) Orientation angle difference ( $\theta$ ), eccentricity, aspect ratio, and circularity of nucleus measured from three different conditions Glass (left column), RA pattern (middle column), RB pattern (right column). The asterisk marks significance $(p<0.001)$ difference when compared with WT cells grown on the glass. $n=27,32,52,21$ cells for glass from one experiment, $n=70,41,48,55$ cells for RA in three independent experiment, $n=41$, $37,52,36$ cells for RB in two independent experiment. ${ }^{*} \mathrm{p}<0.05,{ }^{* *} \mathrm{p}<0.01,{ }^{* * *} \mathrm{p}<0.001$, Wilcoxon based Mann-Whitney $U$ test were performed for statistical testing.

Figure 2. Nuclei of cells with lamin mutations are less resistant to cyclic stretch. Blue, green and red channels in images denote DAPI, GFP-Lamin A transfection and Phalloidin staining in C2C12 cells with wild type (WT) and mutant Lamin A/C (WT, K97E, E161K, R190W). Cells were either (A) grown on PDMS and fixed - without imparting cyclic stretch or (B) grown, imparted cyclic stretch $10 \%$ cyclic stretched with $2 \mathrm{~Hz}$ frequency for $2.5 \mathrm{~h}$.) and subsequently fixed. Scale, $10 \mu \mathrm{m}$. The arrow shows the direction of stretching. (C) Orientation angle difference $(\theta)$, eccentricity, aspect ratio, and circularity of nucleus measured from unstretched and stretched conditions. $n=26,80,72,119$ cells for unstretched and $n=77,55,77,123$ cells for stretched condition in three independent experiment. ${ }^{*} \mathrm{p}<0.05^{*} \mathrm{p}<0.01,{ }^{* *} \mathrm{p}<0.001$, Wilcoxon based Mann-Whitney U test were performed for statistical testing.

Figure 3. Reduction in the lamina thickness due to laminopathic mutation. eGFP-lamin A (containing wild-type and R190W, E161K \& K97E mutations) transfected cells were grown on RA (A) and RB (C) patterned surface. The lower panels of $A$ and $C$ are representing linearization of the nuclear lamina for wild-type and mutant nuclei. The representative FWHMs of the intensity profiles 
Nuclear deformations in cardiomyopathic nuclei

of white the line across the linearized lamina (after Gaussian Fittings) are shown in panel B for RA and in panel D for RB surface. The box plots in the panel B and D represent the FWHM (thickness) of the lamina for RA and RB micropatterned surface respectively. Each dot in the box plot denotes the raw data obtained from each nucleus. The scale bar is $5 \mu \mathrm{m}$. ${ }^{* * *} \mathrm{p}<0.001,{ }^{* *} \mathrm{p}<0.05$, ns $=$ not significant.

Figure 4. Model for lamin meshwork in the presence of mechanical cue in laminopathic cells.

In presence of the external mechanical cue, wild-type lamin A form dense meshwork inside the nucleoplasm and width of the lamina also increases. In the case of laminopathic nuclei, lamin $\mathrm{A}$ meshwork density decreases and the lamina also shows thinning behavior in the presence of the same external mechanical force. The nuclear axis also shows large impairment along the acting stress fiber compared to the wild-type nucleus. 
bioRxiv preprint doi: https://doi.org/10.1101/611665; this version posted April 16, 2019. The copyright holder for this preprint (which was not certified by peer review) is the author/funder. All rights reserved. No reuse allowed without permission.

Nuclear deformations in cardiomyopathic nuclei

\section{Figure 1}

A

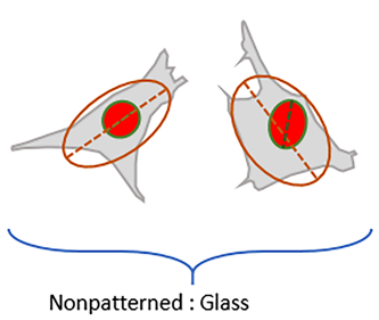

B
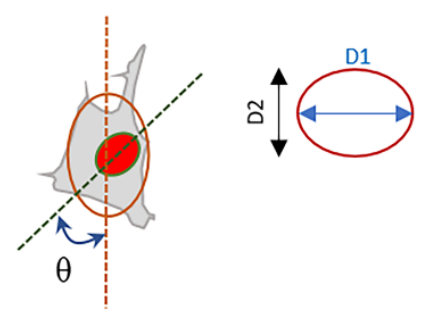

$\mathrm{C}$

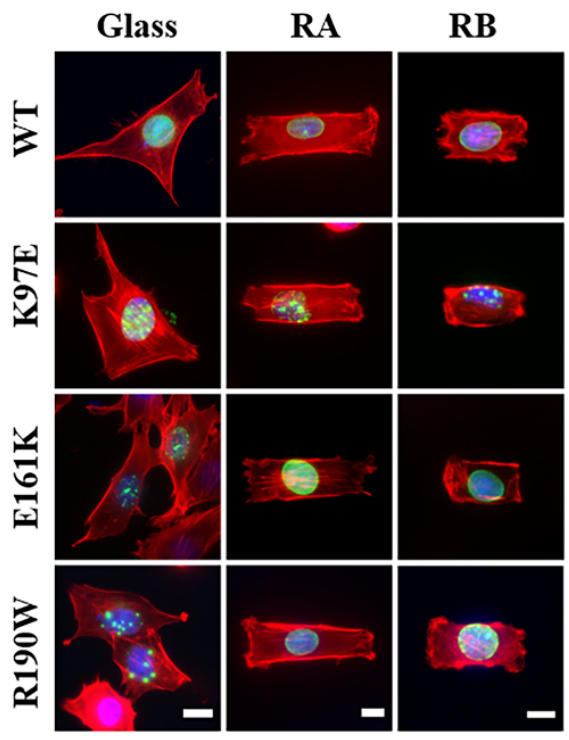

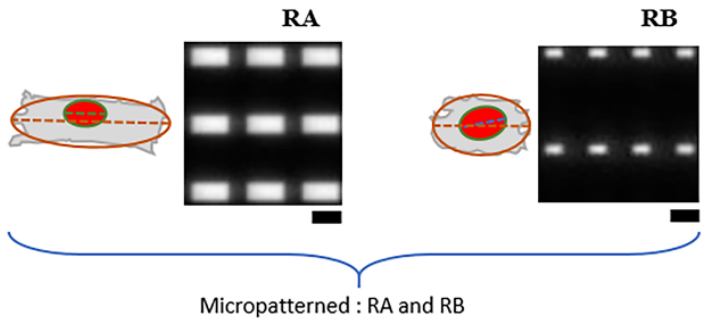

$\mathrm{D}$
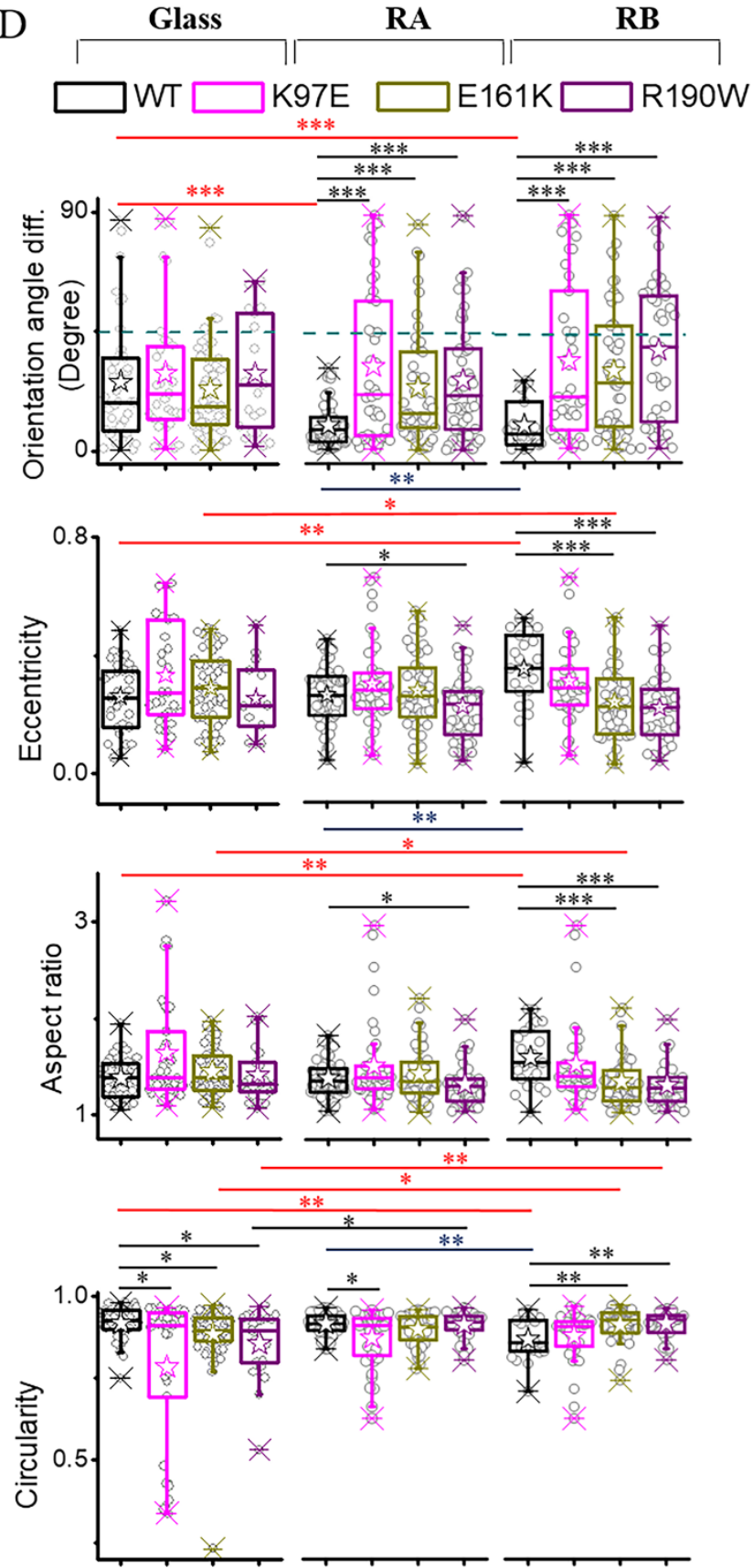
Nuclear deformations in cardiomyopathic nuclei

\section{Figure 2}

A

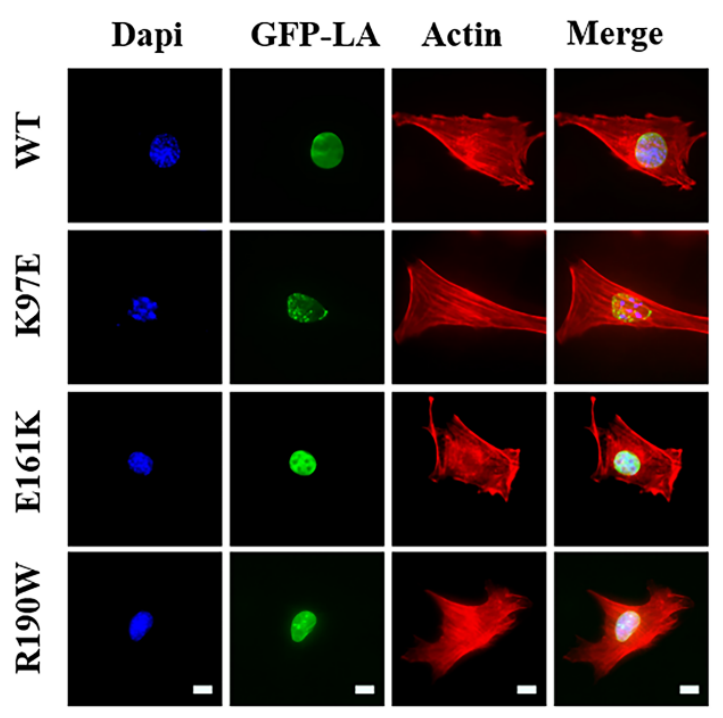

B

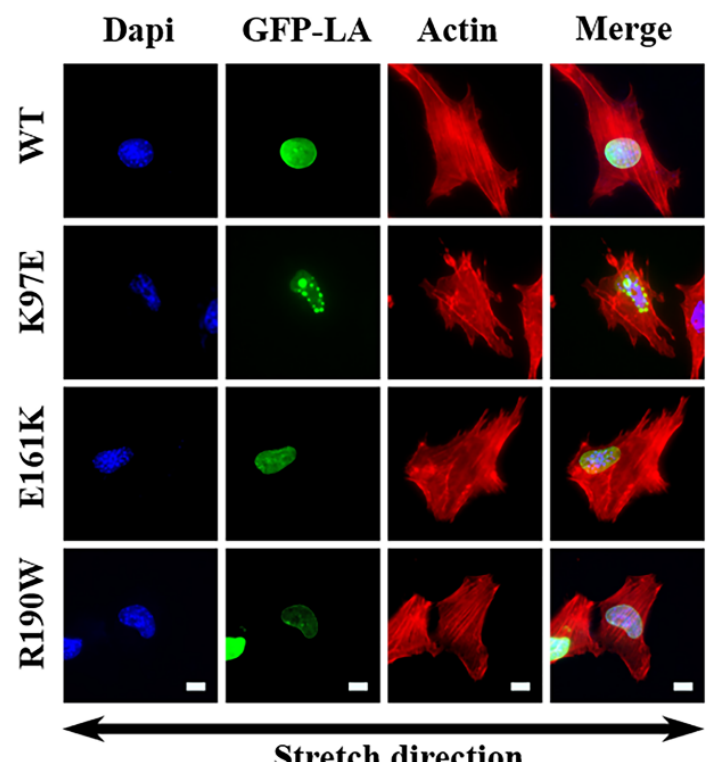

$\mathrm{C}$
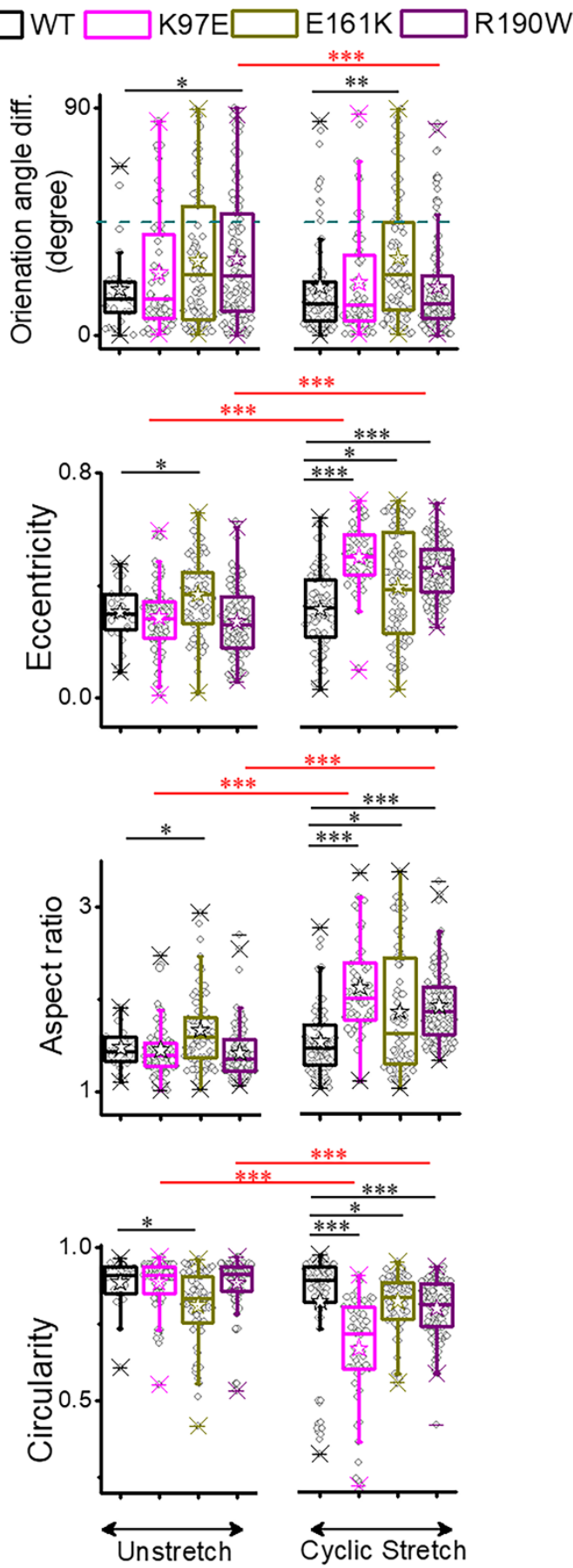
Nuclear deformations in cardiomyopathic nuclei

Figure 3
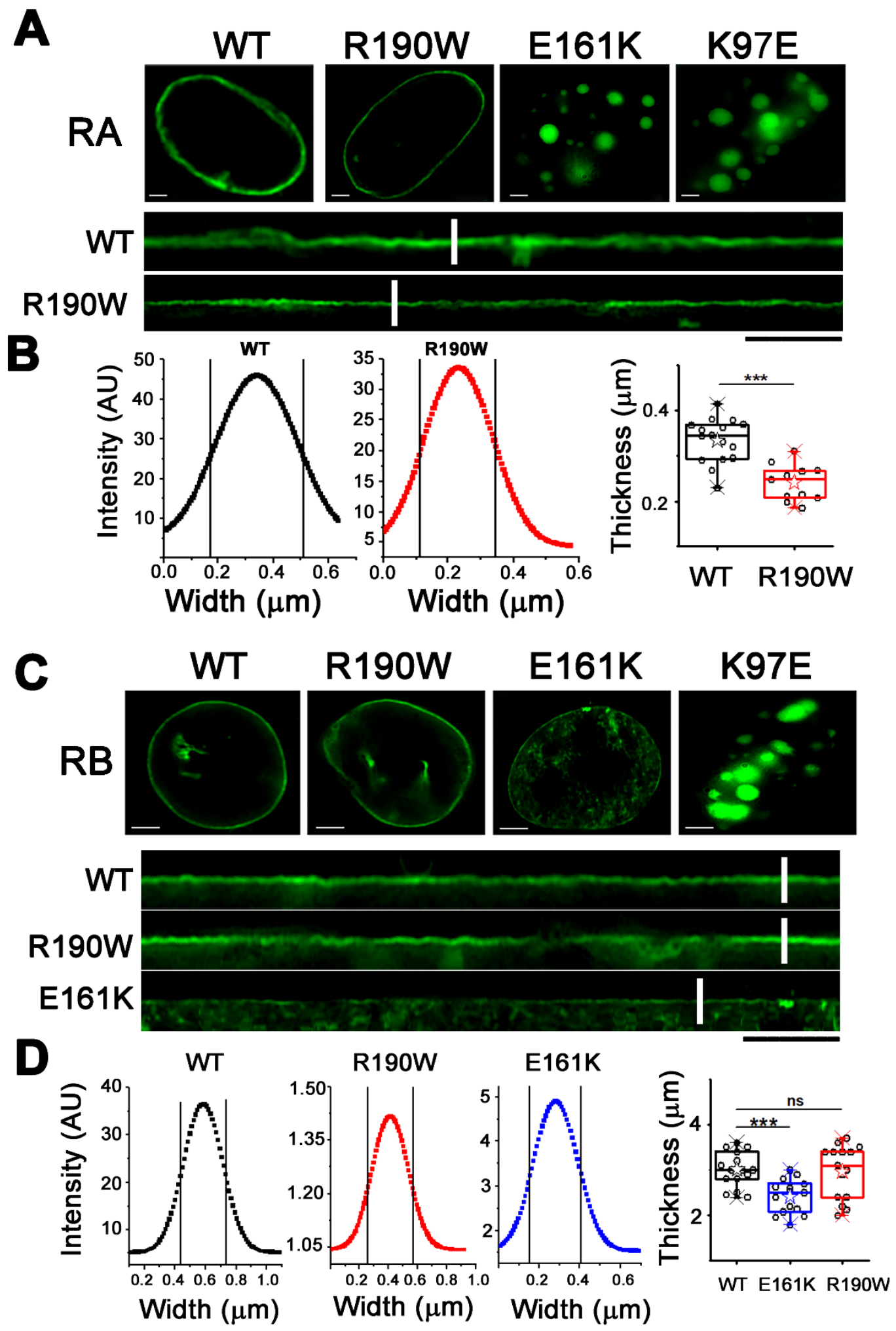
Nuclear deformations in cardiomyopathic nuclei

\section{Figure 4}

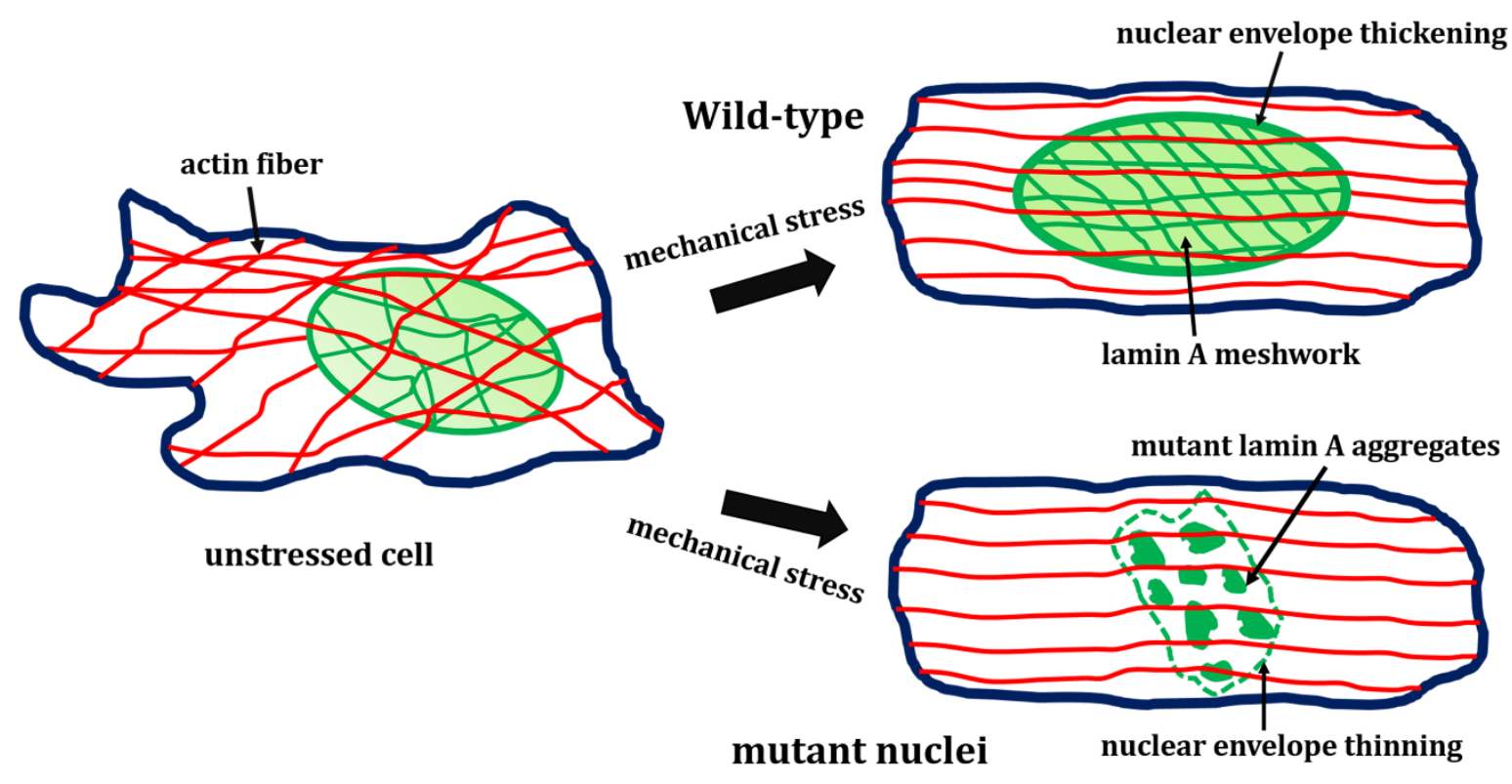

